\title{
Psicología Comunitaria: prácticas en Valparaíso y visión disciplinar de los académicos nacionales*
}

\author{
Héctor Berroeta Torres \\ Universidad de Valparaíso, Valparaíso, Chile. Email hector.berroeta@uv.cl \\ Fuad Hatibovic Diaz \\ Universidad de Valparaíso, Valparaíso, Chile. Email fuad.hatibovic@uv.cl \\ Domingo Asún Salazar \\ Universidad de Valparaíso, Valparaíso, Chile. Email domingo.asun@uv.cl
}

\begin{abstract}
Resumen: Este trabajo analiza las características de las prácticas de intervención y el desarrollo disciplinar de la Psicología Comunitaria en el país. El estudio se organizó a partir de los antecedentes aportados por distintas investigaciones que plantean la existencia de un desfase entre el marco disciplinar y las practicas de intervención. La investigación se llevó a cabo en una muestra de psicólogos de la Provincia de Valparaíso ( $\mathrm{N}=51$ ), quienes respondieron a un cuestionario y luego una sub muestra $(\mathrm{N}=5)$ que fueron entrevistados. Además, se aplicó una entrevista abierta a 8 académicos nacionales de reconocida trayectoria en la disciplina. Los resultados confirmaron en su conjunto que los contenidos del desfase se han agudizado, priorizando por intervenciones individuales centradas en la mejora contingente de las condiciones de vida y salud mental de los participantes. El trabajo concluye que es necesario repensar los contenidos y objetivos de la disciplina en el contexto nacional para retomar el énfasis en la transformación social que es consustancial a la disciplina.
\end{abstract}

Palabras claves: Historia psicología comunitaria, Psicología Comunitaria en Chile, practicas de Intervención.

\section{Community psychology: practice in Valparaiso and disciplinary vision of the nationals academics}

\begin{abstract}
The following essay is the result of a research that analyzes the characteristics of intervention practices and disciplinary development of Communitarian Psychology in the country. The work was based on different studies that suggest there is a gap between the disciplinary framework and the intervention practices.

A survey was applied on a sample of fifty-one psychologists that work in community intervention in the Valparaíso Province, and later, a subsample of five of these psychologists and eight acknowledged national academics were interviewed. Results confirm that the contents of the gap have worsened, giving priority to individual interventions focused on the improvement of life conditions and mental health of the participants. The research concludes that it is necessary to reconsider the contents and objectives of the discipline in the national context, in order to emphasize the social transformation that is inherent to it.
\end{abstract}

Key words: History of communitarian psychology, Communitarian Psychology in Chile, intervention practice.

\section{Psicologia Comunitária: Práticas em Valparaiso e visão da disciplina acadêmica nacional}

Resumo: Este trabalho trata de uma investigação, que procura analisar as características das práticas de intervenção e de desenvolvimento da disciplina de Psicologia Comunitária no Chile.

$\mathrm{O}$ estudo organizou-se a partir de antecedentes provenientes diversos estudos que delineiam(estabelecem) a existência de um desfasamento entre o enquadramento disciplinar e as praticas de intervenção.

A investigação foi realizada a partir de numa amostragem de psicólogos que intervêm na comunidade da Província de Valparaíso, dos quais 51 responderam a um questionário e 5 desses profissionais responderam a uma entrevista. Realizou-se também uma entrevista aberta a oito académicos chilenos, de renome no âmbito da disciplina. Os resultados confirmaram no seu conjunto que os conteúdos de desfazamento se agudizaram, dando prioridade às intervenções individuais que se centram na melhoria das condições de vida e saúde mental dos participantes. O trabalho conclui que é necessário repensar os conteúdos e objectivos da disciplina no contexto nacional, para recolocar a ênfase na transformação social que é consubstancial à disciplina.

Palavras-Chave: História Psicologia Comunitária, Psicologia Comunitária em Chile, Praticas de Intervenção 


\section{Presentación}

En Chile la Psicología Comunitaria ha pasado por distintos momentos dependiendo del contexto sociopolítico y los respectivos marcos institucionales derivados de las políticas institucionales. En los años '80 la intervención se realiza principalmente desde las Organizaciones no gubernamentales en prácticas fuera de la institucionalidad gubernamental y centradas principalmente en la lucha por la reconstrucción democrática; en los '90 estas prácticas se incorporaron progresivamente a la nueva institucionalidad gubernamental de los primeros gobiernos democráticos y las ONGs se reconvirtieron con la pérdida del financiamiento externo en organismos ejecutores de política social estatal (Krause y Jaramillo, 1998). Al terminar esta década se produce un giro en las formas de hacer intervención, que pone de manifiesto una disociación progresiva entre las prácticas psicológico-comunitarias y el marco teórico-conceptual que las sustenta. Durante los 2000 se consolida y tecnifica por parte del estado un enfoque de habilitación individual en el abordaje de los problemas y la integración social, dejando en segundo plano las iniciativas de tipo territorial.

En este contexto de cambio, en el campo de la intervención social en el país, la Psicología Comunitaria en Chile ha experimentado un proceso de mutación tanto en sus énfasis como en sus modalidades de trabajo; su noción de transformación que le es constitutiva y que ha dado sustento a los principales desarrollos teóricos de la disciplina impartidos en la formación profesional están variando para adecuarse a las demandas que se presentan en las prácticas de intervención que se realizan.

En este marco, la presente investigación se propuso actualizar la información acerca de las características de las acciones interventivas desarrolladas por los propios psicólogos en las comunidades y describir la visión académica acerca del desarrollo de la disciplina y sus tensiones. En concreto se planteo conocer las concepciones teóricas, las estrategias de intervención y las evaluaciones que realizan los psicólogos que trabajan en comunidad en la provincia de Valparaíso y explorar la visión de los principales académicos chilenos en la disciplina.

\section{Orígenes y principios disciplinares}

El inicio formal de la psicología comunitaria como término, disciplina y campo profesional es la Conferencia de Swampscott, Massachussets titulada "Conference on the Education of Psychologists for Community Mental Health”, realizada el año 1965 en Boston (Tyler, 1984; Montero, 1994; Alfaro, 1993). En ella se define el rol del psicólogo comunitario como un "agente de cambio social y conceptualizador participante en las transformaciones comunitarias" (Aguilar \& Cruz, 2002. P, 56), que se adscribe a un "modelo interdisciplinario, entre las ciencias sociales y la psicología, a un campo de acción diferente, entre los individuos y la sociedad, y al cambio social planeado" (Tyler, 1984).

En el caso de América Latina, recién en la década de los setenta se puede hablar de una Psicología Comunitaria incorporada al trabajo con comunidades. Sin embargo, sus orígenes se remontan a la crítica de los años 50' que profesionales y académicos vinculados a las Ciencias Sociales plantearon a los enfoques teóricos dominantes y las prácticas que les sucedían, en donde se releva la necesidad de redireccionar la investigación y la intervención hacia la generación de un cambio social.

La idea central de la disciplina es trabajar en, con y para la comunidad, lo que significa redefinir el objeto y el método, revisar la teoría, y reestructurar el rol de los profesionales de la psicología (Montero, 1994). Su objeto es el estudio de los factores psicosociales que permiten desarrollar, fomentar y mantener el control y el poder que los individuos pueden ejercer sobre su ambiente individual y social, para solucionar problemas y modificar cambios en los ambientes y en la estructura social. (Montero, 1984). Las principales características de la psicología social comunitaria, según la revisión de diversos autores realizada por Montenegro (2001) son: 
- Énfasis en la transformación. El objetivo primordial de la acción comunitaria es la transformación de la realidad social, a través de procesos de reflexión-acción.

- Los problemas sociales son causados por estructura social desigual, en la cual la inmensa mayoría de las personas está excluida de los recursos que por derecho le corresponden.

- Uso de las teorías de la ideología, la alienación y el poder entre otras herramientas psicosociales de comprensión e intervención.

- Construccionismo social, esto implica que el conocimiento se da en los intercambios sociales y que es necesario comprometerse con el desarrollo de modelos y métodos que permitan la libre expresión de diferentes construcciones de las personas investigadas.

- Problematización entre la producción teórica y la aplicación del conocimiento. Se parte del presupuesto de que el conocimiento se produce en la interacción entre el profesional y los sujetos de investigación.

- Imposibilidad de la neutralidad del investigador. Por lo tanto, el agente externo debe explicitar su compromiso con los miembros de la comunidad.

- Relación horizontal entre quienes intervienen y quienes son intervenidos

Montero (2004) plantea que los principios de la psicología social comunitaria se encuentran contenidos en lo que denomina el paradigma de la construcción y transformación critica. Este paradigma se estructura en torno a 5 productos: ontológico, que reconoce el carácter productor de conocimiento de los miembros de la comunidad; epistemológico que plantea la construcción social del conocimiento; metodológico que fomenta formas de intervención participativas, una ética de la relación centrada en el bien común y política orientada a la liberación.

\section{Trayectoria y tensiones de la Psicología Comunitaria en Chile}

En sus comienzos el movimiento de la PC nacional, identifica como uno de sus valores el hacer Psicología en forma más equitativa y adecuada a los recursos del país y prescribe al psicólogo un rol de agente de cambio social (Asún y Krause, 1995). Este inicio se sitúa a fines de los años '50 y principios de los '60, desarrollándose experiencias de trabajo a partir del marco de abordaje comunitario de la salud mental, que se concreta en los programas de trabajo de la Psiquiatría Intracomunitaria y la Salud Mental Poblacional (Alfaro; 1993).

Con el golpe militar de 1973 y sus consecuencias sociopolíticas, se produce un quiebre en la evolución de la PC Chilena, reapareciendo durante los años 80, ligada a un proyecto político. Este resurgimiento se produce en el contexto de la dictadura militar como una propuesta alternativa, una expresión de esperanzas colectivas y de una práctica profesional que intentó zafarse de métodos y modelos tradicionales, insertándose en la vida concreta de las personas. Con la llegada de la democracia en los 90 se supone un cambio en la concepción institucionalizada de la atención social, la cual comenzó a ser considerada un derecho de los ciudadanos. Los servicios de atención social fueron concebidos como instrumentos económicos, técnicos y humanos de los cuales se dota una sociedad para promover las condiciones que facilitan la libertad y la igualdad de los ciudadanos, así como también el progreso social y económico (Rozas, 1994-1995 en Krause, 1998).

El desarrollo de la psicología comunitaria en el país, principalmente durante la transición política, ha estado fuertemente determinado por su relación con las políticas sociales. Como señala Alfaro (2007 p.44) "las orientaciones de las políticas sociales establecen el marco sociopolítico y de relaciones sociales desde donde, de manera principal (pero no única) estas prácticas son configuradas y organizadas, constituyendo por tanto el marco de condiciones de posibilidad para el desarrollo de las prácticas de intervención comunitarias". Por tanto, esta influencia del contexto socio-institucional en el desarrollo de las prácticas de intervención comunitaria, genera un progresivo proceso de institucionalización disciplinar que distancia a la psicología comunitaria nacional de los principios constituyentes del paradigma de la transformaron social.

Un primer antecedente en esta dirección y fundamental para comprender el estado de aplicación de la PC y su relación con el contexto institucional de la política social, en el último tercio de los años 90, es el estudio «Intervenciones psicológico comunitarias en Santiago de Chile» realizado por Krause y Jaramillo 
(1998). En este estudio se investigaron las estrategias y formas de gestión de los centros y programas existentes en siete comunas de Santiago de Chile que desarrollan intervenciones psicológico-comunitarias. En este trabajo se muestran con claridad las tensiones que se generan entre los principios de la PC y las prácticas de intervención que se desarrollan en el campo de la psicología comunitaria nacional. En este sentido, las autoras concluyen que la psicología comunitaria nacional se encuentra en un fuerte proceso de institucionalización, donde los énfasis por la transformación y el cambio social propios de la disciplina han dado paso a una acción de orientación gubernamental y académica. Sin embargo, señalan que aún a pesar de su creciente institucionalización mantiene algunos elementos, tales como la búsqueda de la participación de la comunidad y la valoración positiva de su autonomía y poder.

Un segundo antecedente es la investigación "Aproximación al Desarrollo Actual de la Psicología Comunitaria, desde el Análisis de las Prácticas que esta Construye en el Campo de la Intervención Social”, realizado por Saavedra (2005). Se trata de una aproximación cualitativa a las acciones prácticas y las disposiciones representacionales (habitus) en torno a las prácticas de psicología comunitaria presentes en los interventores/profesionales de experiencias comunitarias desarrolladas en Valparaíso y Viña del Mar. Esta investigación entrega una caracterización de las acciones prácticas desarrolladas por los interventores/profesionales, que la autora plantea como el resultado de la relación dialéctica existente entre el sistema de disposiciones (de los profesionales de la psicología comunitaria) y las posibilidades y censuras del entorno (intervención social y políticas sociales). Se rescata la visión de los interventores/profesionales, los cuales describen su rol como un dispositivo o mecanismo que favorece el ajuste del comportamiento de los sujetos al orden social establecido y, por tanto, como una herramienta de la institucionalidad pública o privada para ejercer tal dominio sobre las condiciones de existencia de los sujetos destinatarios. Por otra parte, en relación al espacio de producción académica, los interventores/profesionales plantean un desarrollo escaso de prácticas dialécticas entre teoría y práctica, tendencia que es vista como "tensiones disciplinarias" en el desarrollo actual de la psicología comunitaria, y que se relaciona con los modelos teóricos, las técnicas producidas y el conjunto de prácticas que son el resultado de la articulación de diferentes paradigmas de pensamiento y su mayor o menor acoplamiento con el contexto sociocultural.

Un tercer antecedente, que aporta una aproximación distinta para analizar la tensión entre el quehacer profesional y los marcos teórico-conceptuales en la psicología comunitaria nacional, es el análisis socio histórico de la trayectoria de la disciplina y el contexto socio institucional de las políticas sociales, que ha venido desarrollando Alfaro (2000; 2004; 2007). En su revisión, plantea que si bien la política social se ha orientado a un nuevo enfoque centrado en la vulnerabilidad social, el marco de las estrategias de intervención mantiene su énfasis en la carencia y en la satisfacción de necesidades básicas, sólo cambian los criterios para la asignación de recursos que se amplían y diversifican en función del binomio riesgo/vulnerabilidad. Por tanto señala que la política social continúa orientada a entregar prestaciones individuales; que los programas sociales en general no incorporan procesos participativos en la generación, gestión, desarrollo y evaluación de las intervenciones; y que los técnicos que operan a nivel central, son los únicos actores que inciden realmente en el diseño de los programas sociales (Alfaro, 2007).

Estos antecedentes, dan cuenta desde distintas posiciones, la existencia de una disociación entre las prácticas psicológico-comunitarias y el marco teórico-conceptual de la Psicología Comunitaria. Esta tensión que claramente se ha profundizado en los últimos años, requiere ser estudiada y reflexionada. Es relevante actualizar y profundizar los análisis sobre estas tensiones desde nuevas perspectivas y actores, de tal forma de evaluar sus actuales características y explorar nuevas miradas que favorezcan la reflexión sobre el desarrollo de la disciplina en el país.

\section{Metodología}

La investigación se estructura como un diseño descriptivo-exploratorio con procedimientos cuantitativos y cualitativos de producción y análisis de información

Se consideró como Población de estudio a todas aquellas experiencias comunitarias del campo de la intervención social desarrolladas en la provincia de Valparaíso, que cumplieran con los siguientes criterios: Planteamiento explícito en los objetivos, de intervenir -directa o indirectamente- sobre el bienestar 
psicológico de las personas; Inserción geográfica en la misma comuna de su población destinataria; Realización de actividades de intervención a nivel grupal o con la comunidad en general, de manera exclusiva o paralela a intervenciones a nivel individual; y la presencia de un profesional psicólogo en la ejecución de sus intervenciones.

A partir de estos criterios se conformó una muestra no probabilística e intencionada de 51 psicólogos (as), 33 pertenecientes a la red Sename, 4 a programas de prevención de drogas a nivel comunal (Previene), 2 de corporaciones municipales de educación, 6 de consultorios de atención primaria, 2 de proyectos sociales de programas Fosis, 1 de proyectos del programa Comuna Segura, 1 de centro de salud mental y psiquiátrico y 2 de oficinas municipales. A estos profesionales se les aplico un Cuestionario de preguntas cerradas, organizado en cinco secciones: (a) Aspectos institucionales; (b) Descripción de usuarios; (c) Información del profesional; (d) Características de la acción profesional; y (e) Formación académica.

A partir del análisis de los resultados obtenidos en el cuestionario general se seleccionó y entrevistó a una sub-muestra de 5 psicólogos (as) que se autodenominaron como "psicólogos(as) comunitarios(as)", los tópicos indagados fueron: Identidad profesional, Características de la Psicología Comunitaria, Noción de Cambio social, Aspectos políticos de la Intervención, Modelos teóricos utilizados y formas de Evaluación, Objetivos y Niveles de la intervención, Característica de la Satisfacción profesional y Percepción de las Políticas Sociales.

Por último, se entrevistó a ocho académicos nacionales seleccionados por su reconocida trayectoria en la formación de pre y pos grado en cursos y programas de magíster en el área específica de psicología comunitaria y salud mental en diversas universidades estatales y privadas del país (ver Alfaro y Berroeta, 2007). Las entrevistas tuvieron un formato abierto conducidas según un guión temático -previamente enviado a los entrevistados- que consideraba los siguientes tópicos: (a) Transformaciones de la Psicología Comunitaria, (b) Relación entre Psicología Comunitaria y políticas sociales; (c) Psicología Comunitaria e Intervención Psicosocial; y (d) Las tendencias de futuro para la Psicología Comunitaria en Chile.

\section{Resultados}

\section{Resultados cuantitativos}

\section{Caracterización de los y las profesionales}

Participaron un total de 51 psicólogos, de los cuales un 74,5\% son mujeres y un $23,5 \%$ son hombres., un $72,5 \%$ provienen de Universidades Tradicionales, mientras que un 27,5\% se formaron en Universidades privadas, un $19 \%$ ha cursado programas de magíster, al respecto un $51 \%$ evalúa su formación académica como suficiente, seguido de un 19,6\% que la define como satisfactoria. Las áreas que identifican como deficitaria de su formación son: la sistematización de prácticas sociales $(37,3 \%)$, las herramientas operativas $(19,6 \%)$ y la falta de marcos teóricos $(15,7 \%)$.

Respecto a la autodefinición profesional, sólo un 22\% se definen como psicólogo/a comunitario/a mientras que un $24 \%$ lo hace como psicólogo/a clínico/a o como sólo psicólogo (ver gráfico) 


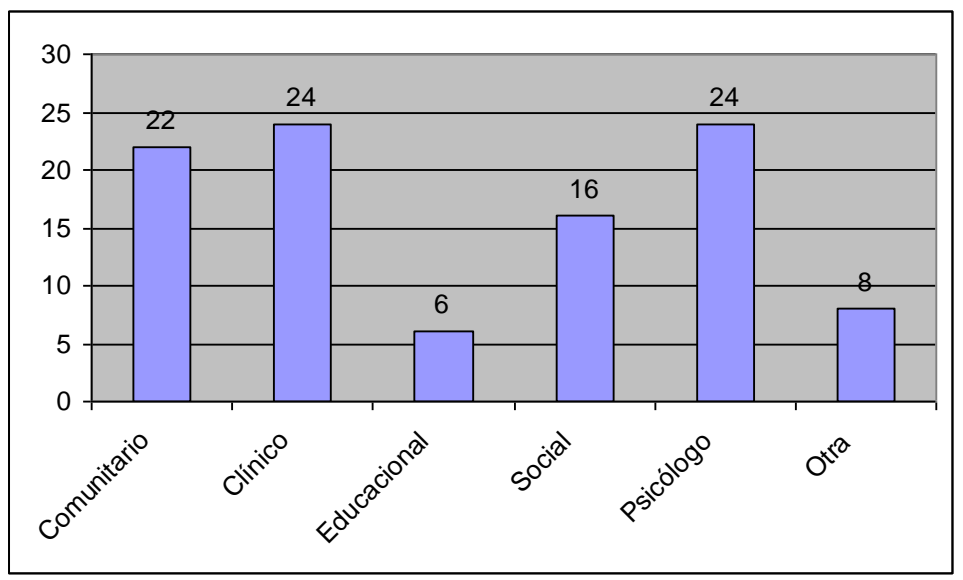

\section{Dimensiones de la intervención}

El $92,2 \%$ de los participantes describe a la población usuaria de los programas como pobres e identifican la demanda espontánea como la principal vía de acceso $(65,3 \%)$.

Respecto a las modalidades de intervención que se realizan, destaca que un $64,7 \%$ de los psicólogos(as) realizan su intervención de modo preferente en el mismo centro, superando por amplio margen a quienes intervienen en la casa de los usuarios $(13,7 \%)$ o en la calle $(2 \%)$. El foco de intervención se encuentra distribuido equilibradamente entre: promoción $(35,3)$, prevención $(31,4 \%)$ y tratamiento $(27,5 \%)$.

Las estrategias de intervención preferentes son los talleres y la psicoterapia, con un 31,4\% y un $25,5 \%$ respectivamente, seguido por las actividades de capacitación $(17,6 \%)$ y la intervención en crisis $(9,8 \%)$. En relación al nivel en que se realizan las intervenciones, la mayoría de los psicólogos(as) interviene a nivel individual con un $49 \%$, seguido del $33,3 \%$ en el nivel grupal y sólo un 9,8\% lo hace a nivel comunitario.

Por último, respecto a la participación de los usuarios en las distintas etapas del proceso de intervención, un $48 \%$ la circunscribe al diagnóstico y un $6 \%$ a la fase de evaluación.

\section{Índice de Prácticas comunitarias}

Uno de los propósitos centrales de esta investigación es dar cuenta de un modo integral de las prácticas interventivas de los psicólogos y psicólogas que intervienen en comunidad. Como una forma de explorar en estas prácticas, se indagó en diferentes dimensiones, descritas anteriormente, las cuales se sintetizaron en un índice de "Prácticas comunitarias" en el que se consideraron las siguientes dimensiones: Participación de los usuarios, Lugar en que se realizan las intervenciones, foco de intervención, modalidad de intervención, nivel de intervención, y nivel de participación de los usuarios. El procedimiento de construcción del índice consistió en transformar cada item de la dimensión analizada a un puntaje estándar, en donde se le asignó un valor mayor al item que se aproximara a una concepción más cercana a la psicología comunitaria. Por ejemplo, en la dimensión Lugar en que se realizan las intervenciones, los psicólogos(as) que realicen su intervención en la calle obtendrán un mayor puntaje que aquellos que intervengan en las instalaciones del centro, esto quiere decir que estos últimos estarán más lejanos de una práctica comunitaria ideal, que es la que sintetiza el índice.

En síntesis, los resultados de este índice se agruparon en 3 niveles: bajo, medio y alto, en donde solo un $15,7 \%$ de los psicólogos(as) tendría un alto índice de prácticas comunitarias, es decir, este pequeño grupo es el que exhibe en su intervención una mayor sintonía con el trabajo comunitario, en el sentido que interviene preferentemente fuera de las instalaciones del centro, privilegia un nivel de intervención comunitario o grupal, su foco de intervención está en la promoción, y considera la participación de los usuarios en distintos momentos de la intervención. 

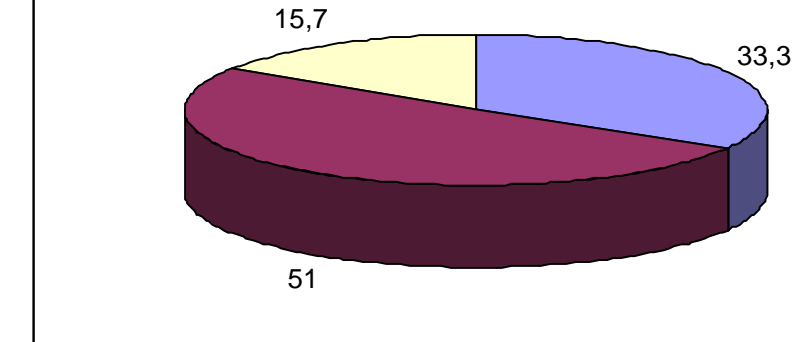

El 51\% de los psicólogos(as) se agrupan en un nivel medio, donde la práctica comunitaria tiene alguna presencia, pero no hegemoniza la intervención, es decir, es un grupo en que su intervención se centra en un nivel grupal, con alguna presencia de lo individual; interviene en las instalaciones del centro en forma preferente; su foco de intervención está en la prevención; y considera la participación de los usuarios en las intervenciones, aunque tienen una menor presencia que en el grupo con un alto índice.

Por último, se encuentra el 33,3 \% de los psicólogos(as) que se agrupa en el nivel bajo, este grupo se caracteriza por tener una práctica interventiva alejada del ideal de intervención comunitaria, este grupo se caracteriza por realizar sus intervenciones en el centro institucional, privilegiar un nivel de intervención individual, focalizar su trabajo en el tratamiento, plantear la psicoterapia como su modalidad de intervención preferente y tener una baja participación de los usuarios en sus intervenciones.

\section{Análisis cualitativo}

En el proceso de análisis de las entrevistas se identificaron las unidades mínimas de información y las unidades de contexto, y posteriormente se aplicó un esquema de categorización, del cual surgieron las siguientes categorías: Concepciones Teóricas y Estrategias de Intervención; La Política Social y su Relación con las Prácticas de Intervención; El Espacio de Formación académica y Desafíos y Proyecciones de la Psicología Comunitaria en Chile. A continuación se presenta la descripción de estas categorías:

\section{Concepciones Teóricas y Estrategias de Intervención}

Los profesionales al caracterizar lo comunitario lo hacen como un enfoque que comprende los problemas sociales desde una aproximación grupal, con énfasis en las comunidades y los grupos humanos. Mientras que a la Psicología Comunitaria la describen como una perspectiva que se nutre de otras áreas o subáreas de la psicología en general; orientada al ejercicio de los derechos humanos; que comprende el fenómeno individual como parte de un contexto de dinámicas y relaciones grupales; que intenta mejorar la calidad de vida y favorecer la transformación o el cambio de la realidad; y que busca generar procesos de autogestión por parte de los colectivos vulnerados o excluidos como estrategia para alcanzar la integración social.

Las apreciaciones que los profesionales tienen de los modelos teóricos que sustentan su intervención, dan cuenta de una poca claridad conceptual y una cierta distancia de los referentes teóricos. Perciben a las teorías como alejadas de la realidad concreta en que trabajan.

Estas características que se observan en el desempeño de la labor profesional de los psicólogos que trabajan en comunidad, dan cuenta del marcado alejamiento de los modos tradicionales de entender la psicología comunitaria en el país. 
"La verdad es que nosotros en general trabajamos acá en equipo y nos manejamos poco en las teorías (...) De repente las teorías son súper alejadas de lo concreto, aquí nosotros trabajamos en una situación súper concreta, real, aterrizada que en la teoría." (Profesional 2)

Los expertos académicos señalan que los referentes teóricos que sirvieron de sustento para el desarrollo histórico de la psicología comunitaria latinoamericana, han perdido vigencia y aplicabilidad en el contexto actual, ya que estos obedecían a un proyecto de sociedad y una forma de enfocar la transformación social relacionada con visiones político-ideológicas contingentes a un momento histórico.

"Hay ciertas conceptualizaciones que siguen siendo interesantes. Pero muchas de ellas se dieron en un marco ideológico y en una apuesta a un proyecto social determinado, y ese proyecto no se concretó... hay reestructuraciones que hacer porque esas categorías comenzaron a quedar estrechas para explicar los nuevos fenómenos" (Académico 1)

En este sentido plantean que el gran desafío para la psicología comunitaria nacional es la utilización y construcción de marcos teóricos y conceptuales que sean más pertinentes a la realidad actual. El paradigma construccionista se presenta como una posibilidad para enmarcar esta nueva forma de concebir la psicología comunitaria.

"Tal vez el elemento clave de toda esta dinámica sea el tema del constructivismo y el construccionismo. Los psicólogos comunitarios estamos entendiendo que dicho paradigma es parte de nuestra esencia, pero, pese a que somos eso, debemos entender mejor de qué se trata y tomar conciencia de que existe una forma más apropiada de concebir la realidad, una forma asociada de hacer investigación e intervención" (Académico 4)

\section{La Política Social y su Relación con las Prácticas de Intervención:}

Todos los psicólogos que han participado del estudio se encuentran adscritos a la ejecución de alguna política social, sean éstas dependientes de servicios públicos, ministeriales o de reparticiones municipales. No fueron identificadas intervenciones independientes que cumplieran con los criterios de selección.

Se ha constatado que el nivel de involucramiento que ha alcanzado la política pública en el ejercicio de la labor profesional de los psicólogos que intervienen en comunidad, es tal, que existe una suerte de naturalización de ciertos mecanismos diseñados para la ejecución de las líneas programáticas. Los profesionales remiten sus funciones a elaborar, ejecutar y evaluar proyectos; y le asignan a la conformación de la dupla psicosocial la capacidad de ampliar la mirada de los fenómenos sociales. De este modo, se distancian de las perspectivas desprofesionalizantes propias del enfoque social comunitario.

"A ver... hay un... en el diseño de las propuestas de proyectos, tiene que ver con la elaboración de proyectos, o sea elaboración, ejecución y evaluación, pero un tema de diseño en la parte de ejecución, también hay en este último tiempo, se ha potenciado harto el tema de dupla psicosocial, ser parte de dupla psicosocial, entonces hay un rol ahí, que para mi igual ha sido medio novedoso, como de tratar de aportar desde cosas, por así decirlo, más bien psicológicas, pero puestas al análisis de un compañero(a), que es tu contraparte técnica" (Profesional 4 )

Los objetivos de su accionar están dados explícitamente por la planificación de los proyectos en los cuales participan, reconocen que las políticas sociales enmarcan sus intervenciones, aunque en muchos casos señalan que brinda autonomía al interventor, plantean que se produce una tensión entre la lógica tecnócrata y burocrática de las políticas sociales y ciertas dinámicas de participación que requiere los enfoques comunitarios. Principalmente en orden a abordar aquellos objetivos relacionados con el protagonismo de los beneficiarios en el logro de los cambios o transformaciones de su propia realidad.

Para los académicos, las prácticas de la psicología comunitaria a partir de los años noventa, no pueden descontextualizarse del escenario político social que comienza a vivir el país, como es el lento retorno a la democracia. En esta época, el marco de referencia para las prácticas de intervención son las políticas sociales, 
que transforman en buena medida a la Psicología Comunitaria en un instrumento para la resolución de los problemas sociales definidos y priorizados por la política pública.

Los académicos señalan que las políticas sociales condicionan las prácticas de intervención de los psicólogos(as) que intervienen en comunidad. Esto, debido a que las prácticas consideradas en los programas sociales están sobre planificadas y centradas en la intervención, de modo que no interesa "pensarse" un modelo de sociedad, en donde el interventor se encuentra supeditado a la operatoria del programa, actuando entre limitantes presupuestarias y de planificación. En este proceso se describe un sentimiento de desaliento en el marco de cumplimientos de corto plazo. Existe una centralización de la construcción de problemas sociales sin la incorporación, o esta es muy leve, de la comunidad en la definición de problemas. La visión de los problemas sociales se trabaja sobre un enfoque individual y sin entenderlos en un contexto comunitario. Dependiendo del énfasis de las políticas se espera que se integre la concepción de la colectividad y las dimensiones de la participación en el trabajo comunitario.

"No es concebible, hasta ahora, una Psicología Comunitaria o una Intervención Psicosocial al margen de las políticas sociales. Las prácticas de los psicólogos insertas en los programas sociales están, por definición, condicionadas por las políticas sociales y estas últimas no solamente son una posibilidad, sino que son el contexto del quehacer de estas prácticas" (Académico 6)

A partir de los 90 se asocia el desarrollo de la psicología comunitaria casi exclusivamente con la evolución de las políticas sociales, por tanto estas últimas se constituirán en el marco que permite la evolución de la práctica psicológica comunitaria, pero también va a constreñir fuertemente su accionar.

"Y lo hemos dicho en otras ocasiones: la política social es, sin duda y al mismo tiempo, una posibilidad y una restricción para la Psicología Comunitaria" (Académico 6)

Sin embargo, para algunos académicos, a pesar de lo restrictivo que puede ser para los psicológicos comunitarios desempeñarse desde la política pública, la intervención ha experimentado cambios, es decir se ha desplazado desde enfoques individualistas, hacia enfoques más vinculados a lo territorial y comunitario.

"Recientemente hay mejores condiciones para la Psicología Comunitaria. Justamente en esta línea que estoy planteando, la propuesta gubernamental se da en lógicas más territorializadas, más controladas por el alcalde, por la población a través del voto y, en definitiva, de manera más directa. Por lo tanto, todo eso favorece la emergencia de la comunidad... Y por lo mismo, cualquier profesión que trabaje con la comunidad calza y se ajusta mucho mejor" (Académico 4)

En relación con la noción de cambio social, enfatizada en la tradición de la Psicología Comunitaria Latinoamericana, se podría decir que en Chile esta noción, no le pertenece a las relaciones sociales. El cambio social como cambio de estructura social no tiene presencia en el país.

"Hoy, en las prácticas que realizamos en el país, creo que ha perdido centralidad el uso de una noción de cambio desde las relaciones sociales; a lo más, se ha dado relevancia a las estructuras culturales, en la última generación de programas de intervención". (Académico 7)

Para los académicos los efectos de la política social sobre la participación y las estrategias de intervención, se plantean fundamentalmente desde la afirmación que la psicología comunitaria no surge desde lo popular, sino desde lo institucional. En este contexto, se señala que no hay pensamiento en torno a la participación social, tampoco en control social ni en aspectos culturales, identidad y construcción de sujeto. En el plano de las intervenciones no hay presencia de dinámicas de accionar en torno a la participación de la comunidad en la construcción de problemas. Estos son definidos desde fuera de la comunidad y se focalizan en la carencia de los sujetos. Por último, concuerdan en vincular el desarrollo futuro de la psicología comunitaria con la evolución de las políticas sociales en el país, se plantea que éste campo disciplinar estaría ampliando su radio de influencia al interior del mismo Estado, copando áreas de intervención no tradicionales (políticas ambientales y urbanísticas), y adquiriendo una mayor protagonismo en el diseño de las mismas.

\section{El Espacio de Formación académica:}


Para los docentes las prácticas académicas a partir de los noventa, conjuntamente con el aumento de la oferta en psicología, se comienzan paulatinamente a institucionalizar, la asignatura de psicología comunitaria se instala en las mallas curriculares de las principales universidades del país y a partir del año 2000 se desarrollan un conjunto de programas de postgrado.

Las condiciones sociales y políticas generadas a partir de los 90' en el país, influyeron en la construcción del currículo en las universidades que impartían la carrera de psicología, ya que, la mayoría comenzó a incorporar con más o menos fuerza la asignatura "Psicología Comunitaria" en sus planes de estudio. En un contexto que enfatizaba la preocupación por los problemas sociales expresado principalmente por el fuerte rol que comienza a sumir el Estado.

"Y al volver a Chile, en un contexto político distinto, era inconcebible que una universidad que se considerara seria, y una escuela de psicología que se considerara seria, con un currículo que pretende ser más o menos homologable a otros internacionales, no incorporara la Psicología Comunitaria como disciplina". (Académico 3)

Con la consolidación de la Psicología comunitaria en varias de las mallas curriculares de las más importantes universidades del país, a nivel de pregrado, la oferta se extendió hacia el postgrado con el surgimiento de distintos programas de magíster, los cuales se caracterizan por plantear distintos enfoques de lo comunitario Por tanto, se percibe que estos programas están en una etapa de consolidación y ajuste, lo cual marca una distinción con lo que acontece a nivel de pregrado con la disciplina.

"A nivel de pregrado se observa fundamentalmente la consolidación de la disciplina, pero a nivel de magíster está ocurriendo otra cosa, es un proceso que no está terminado, que está construyéndose, que se está elaborando" (Académico 3)

Por otro lado, los y las académicos destacan la disociación que existiría en el trabajo investigativo que realizarían las universidades y las intervenciones comunitarias o sociales desarrolladas principalmente al alero del Estado. En este sentido, el rol que deben desempeñar las instituciones de educación superior es formar profesionales que no solo realicen investigaciones, sino que sean interventores capacitados, para lo cual es fundamental contar con docentes que estén realizando intervenciones frecuentemente.

"Creo que el gran desafío para una institución de educación superior que quiere formar personas que hagan intervención y no solamente investigación, es que tiene que tener en su interior personas que hagan intervención". (Académico 1)

\section{Desafíos y Proyecciones de la Psicología Comunitaria en Chile}

Como ya se señalaba, a partir de los años 90 y consecuentemente con las demandas de abordajes interventivos de los problemas sociales, se van consolidando la formación académica y los planes de estudio en psicología comunitaria e intervención psicosocial. Los académicos señalan que a pesar de que el saber se ha ido tecnificando en cuanto a desarrollos de diseños de proyectos, de marcos lógicos, de planificación, no se ha incorporado una reflexión en torno a las políticas sociales y sus lógicas de intervención. Plantean como desafíos el incorporar nuevos modelos de formación, como perspectivas sistémicas y ecológicas, además de la conformación de espacios de diálogo, investigación y desarrollos formativos en torno a las condiciones de producción en que se desenvuelve la disciplina.

"Creo que podríamos pensar en una Psicología Comunitaria que imponga algunos elementos de política social. Tal como está ahora, altamente centralizada, no lo veo posible y, por lo tanto, lo que tendríamos que tener es una mayor visibilización de la Psicología Comunitaria en una suerte de lobby, frente a los intelectuales que asesoran las políticas sociales de gobierno". (Académico 1)

Las prácticas relacionadas con la política social han constituido un espacio de enriquecimiento, pero que no ha podido cimentarse dadas las limitaciones de enfoques centrados en los objetivos de los programas. Se ha enfatizado en el saber técnico como respuesta a los requerimientos de los programas sociales existentes. 
Se reconoce el carácter incipiente de la disciplina que se estaría potenciando con la aparición de cursos de post-grado con formación especializada en el tema. Se espera que se generen nuevas reflexiones y evaluaciones más específicas de las prácticas y producciones en torno a discusiones conceptuales.

Los ámbitos de acción, que según los expertos, podría abarcar en un futuro la psicología comunitaria, y por consiguiente deberían ser integrados a la formación de los psicólogos, serían variados, ya que podrían ir desde los temas urbanísticos, medioambientales, hasta los temas de la interculturalidad.

"El tema ambiental es un campo de grandes posibilidades de incorporación del Psicólogo Comunitario. Y el último campo que menciono, es el de la interculturalidad; a mí me parece bellísimo, es un área de trabajo, un ámbito que siempre ha estado presente en Chile". (Académico 7)

\section{Discusión}

Los cambios experimentados en el país en estos últimos años han afectado significativamente el quehacer de la psicología comunitaria, así lo demuestran los resultados de esta y otras investigaciones (Krause y Jaramillo, 1998; Saavedra, 2005; Reyes, 2007; Castillo y Winkler, 2010)

La práctica transformadora, reflexionada y generadora de teoría de la que habla Montero (2004b), no emerge de la praxis de los psicólogos que trabajan en la comunidad o al menos en el quehacer institucionalizado de la práctica que se realiza en negociación con el Estado; y en el caso de la V región tampoco se observa una acción alternativa en la que participen psicólogos, esto queda refrendado con los resultados del "índice de prácticas comunitarias" que nos indica que solo un $15,7 \%$ de los profesionales realizan una práctica de intervención vinculada a lo comunitario.

Las descripciones del quehacer comunitario, que reportan tanto los actores profesionales como académicos, son coincidentes con la afirmación de que "muchas intervenciones en el campo de la psicología comunitaria, por bien intencionadas que sean, no alteran las estructuras pues más bien están dirigidas a ayudar a las víctimas. A lo largo de un continuum que va del mejoramiento a la transformación, nuestras acciones contribuyen principalmente a lo primero y sólo periféricamente a lo segundo", (Prilleltensky, 2004. p. 30). Al parecer en el actual contexto del quehacer socioprofesional que aquí se reporta, no estamos siendo capaces de mover la justicia social al primer plano de nuestras preocupaciones (Prilleltensky y Nelson, 1997).

Los resultados que se presentaron, junto a los datos de otras investigaciones (Krause y Jaramillo, 1998; Saavedra, 2005; Reyes, 2007; Castillo y Winkler, 2010), entregan elementos suficientes para constatar que el paradigma de la psicología comunitaria tal cual lo conocemos, no tiene cabida explicita ni fluida en la actual estructura normativa de la política social chilena.

Esta constatación implica preguntarnos si hemos perdido toda posibilidad, en el marco del quehacer gubernamental, de aspirar a una psicología que como señala Montero contribuya a desarrollar ciudadanos conscientes de sus deberes y de sus derechos, así como a reflexionar críticamente sobre sus actos y sobre los resultados de los mismos, sobre sus motivaciones y sobre sus compromisos, contribuyendo a la transformación de las sociedades, fijando rumbo hacia formas de desarrollo ecológicamente viables y humanamente deseables" (Montero, 2004b.p.28), por tanto, ¿debemos abandonar todo quehacer vinculado a la estructura de la política gubernamental si queremos aspirar a estos idearios? ¿O es posible, como sugiere Prilleltensky (2003), situarnos en una posición intermedia entre la mera ejecución alienante de la lógica programática y el dogmatismo rígido inmovilizante?, es decir, no abandonar las acciones de "mejoría" sino enriquecerlas incorporando el desarrollo sociopolítico y redireccionando las prácticas de intervención.

Al respecto podemos identificar dos direcciones: Una primera asentada en el valor de la institucionalidad democrática, desde el cual se puede plantear que el valor de la Psicología Comunitaria en la estructura gubernamental es modificar la política social, intentando influir en sus discursos, estrategias y en los criterios con que se definen los problemas sociales, propiciando miradas críticas y desarrollando procesos de fortalecimiento de control ciudadano. 
Una segunda, desde una lectura situada, que sostiene que toda acción comunitaria instituye un proceso de articulación, es decir, una práctica que establece relaciones entre elementos o agentes de manera que las identidades de estos son modificadas como resultado de la práctica articulatoria, proceso en el que los sujetos desarrollan acciones que modifican sus entornos y a su vez son modificados por ellos (Montenegro 2002). Por lo cual el agente comunitario, sea cual sea su procedencia, se ve afectado por la acción de los otros y del entorno, desde ahí por tanto, es posible pensar la creación de articulaciones temporales que posibiliten acciones evaluadas como pertinentes por los miembros de las comunidades y que busquen modificar sus espacios de poder y sus condiciones de vida.

Sin embargo no podemos desconocer una postura contrapuesta a esta posición intermedia, de respuesta negativa a las interrogantes planteadas, que entiende a la psicología comunitaria como un campo disciplinar y no como un ámbito de aplicación, por tanto, que sitúa las posibilidades de la acción comunitaria sólo en el contexto de ciertas condiciones y en base a determinados principios valóricos, marco en el cual la dependencia gubernamental es incompatible.

Estas alternativas y otras que probablemente no alcanzamos a vislumbrar, nos plantean la necesidad de continuar con este tipo de investigaciones. Cartografiar el quehacer socioprofesional es una tarea que nos acerca a conocer los alcances y sentidos de un campo que hasta ahora, se muestra borroso y poco delimitado. Es urgente responder la pregunta, ¿Qué es la psicología comunitaria en chile?

\section{Nota}

\footnotetext{
" Articulo basado en el Proyecto de Investigación Psicología Comunitaria en Chile: Un Estudio de las Tensiones Disciplinares y Las Practicas De Intervención Financiado por la Dirección de Investigación de la Universidad de Valparaíso
}

\section{Bibliografía}

Aceituno, R. (1993). La Psicología Comunitaria en Chile. Proposiciones para una intervención ideológica, elementos para una discusión. En R. Olave y L. Zambrano (Eds.). Psicología Comunitaria y Salud Mental en Chile (pp. 32-34). Universidad Diego Portales. Santiago de Chile.

Alfaro, J. (1993). Elementos para una definición de la Psicología Comunitaria. En Olave, R. M. y Zambrano, L. (Comp.), Psicología Comunitaria y Salud mental en Chile (pp. 14-31). Universidad Diego Portales. Santiago.

Alfaro, J. (2000). Discusiones en Psicología Comunitaria. Universidad Diego Portales. Santiago de Chile.

Alfaro, J. (2004). Psicología Comunitaria y Políticas Sociales: Análisis de su desarrollo en Chile, Revista de Psicología. Universidad Bolivariana. Año 1 No 1-2, 2004.

Alfaro, J. (2007). Políticas sociales como condición de posibilidad para el desarrollo de prácticas en Psicología Comunitaria1. En Alfaro, J. y Berroeta, H. (ed.) Trayectoria de la psicología comunitaria en Chile. Prácticas y conceptos. (pp. 43-72) Universidad de Valparaíso. Valparaíso.

Alfaro, J. y Berroeta, H. (ed.) Trayectoria de la psicología comunitaria en Chile. Prácticas y conceptos. Universidad de Valparaíso. Valparaíso.

Asún, D., Krause Jacob, M., Aceituno, R., Alfaro, J. y Morales, G. (1995). La Psicología Comunitaria en Chile Análisis de sus características y perspectivas. En Wiesenfeld, E. y Sánchez, E. (comp.). Psicología Social Comunitaria. Contribuciones Latinoamericanas (pp. 151-188). Tropykos. Caracas.

Cruz, F. y Aguilar, M. (2002). Introducción a la Psicología Comunitaria. CCS. Madrid.

Krause, M. Y Jaramillo, A (1998). Intervenciones Psicológico-Comunitarias en Santiago de Chile. Pontificia Universidad Católica de Chile. Santiago.

Martín-Baró, I. (1989): Sistema, grupo y poder. Psicología social desde Centroamérica II. UCA Editores. San Salvador. 
Montenegro, M. (2001). Conocimientos, Agentes y Articulaciones: Una Mirada Situada A La Intervención Social. Tesis Doctoral, Programa de Doctorat en Psicología Social. Universitat Autónoma de Barcelona.

Montenegro, M. (2002). El Cambio Social Posible: Reflexiones en torno a la intervención social. En ARCIS. Políticas Sujetos y Resistencias. Debates y Críticas de Psicología Social. Cuadernos de Psicología Social N 1. (pp.229-236). Universidad Arcis. Santiago.

Montero, M. (1980). La Psicología Social y el desarrollo de las comunidades en América Latina. Revista Latinoamericana de Psicología, 12, (1), 159-170.

Montero, M. (1994). Vidas Paralelas: Psicología Comunitaria en Latinoamérica y en Estados Unidos. En Montero, M. Psicología Social Comunitaria. Editorial: Universidad de Guadalajara.

Montero, M (1998). La Comunidad Como Objetivo Y Sujeto De La Acción Social”. En Martín Gonzáles. Psicología Comunitaria. Fundamentos y Aplicación. Síntesis. Madrid.

Montero, M., (2004) Introducción a la Psicología Comunitaria. Paidos. Buenos Aires.

Montero, M. (2004b) Relaciones Entre Psicología Social Comunitaria, Psicología Crítica y Psicología de la Liberación: Una Respuesta Latinoamericana. Revista Psykhe, Vol. 13, № 2, 17-28

Prilleltensky, Isaac. (2003) Prólogo. En Montero, Maritza. Introducción a la Psicología Comunitaria. Paidós. Buenos Aires.

Prilleltensky, I. \& Nelson, G. (1997). Community psychology: Reclaiming social justice. En D. Fox \& I. Prilleltensky (Eds.) Critical psychology: An introduction (págs. 166-184) Sage. Londres, Inglaterra.

Rozas, G. (1994-1995). Psicología Comunitaria en el desarrollo local y regional. Revista de Psicología, Universidad de Chile, 5, 47-64.

Saavedra, C. (2005). Aproximación al desarrollo actual de la psicología comunitaria, desde el análisis de las prácticas que ésta construye en el campo de la intervención social. Tesis de Magíster en Psicología Comunitaria. Universidad de Chile.

Tyler, F. (1984).La Psicología Comunitaria y sus implicancias para los países en vías de desarrollo. Revista Latinoamericana de Psicología, 16, (2) Edit. ABC Bogotá 291-307. 